\title{
Self-Similarity of Plasmon Edge Modes on Koch Fractal Antennas
}

Edson P. Bellido, ${ }^{\dagger}$ Gabriel D. Bernasconi, ${ }^{\ddagger}$ David Rossouw, ${ }^{\dagger}$ Jérémy Butet, ${ }^{\ddagger}$ O० Olivier J. F. Martin, ${ }^{\ddagger}$ and Gianluigi A. Botton* ${ }^{\dagger}$

${ }^{\dagger}$ Department of Materials Science and Engineering, McMaster University, 1280 Main Street W., Hamilton, Ontario L8S 4L7, Canada ${ }^{\ddagger}$ Nanophotonics and Metrology Laboratory, École Polytechnique Fédéralede Lausanne, 1015 Lausanne, Switzerland

\section{Supporting Information}

ABSTRACT: We investigate the plasmonic behavior of Koch snowflake fractal geometries and their possible application as broadband optical antennas. Lithographically defined planar silver Koch fractal antennas were fabricated and characterized with high spatial and spectral resolution using electron energy loss spectroscopy. The experimental data are supported by numerical calculations carried out with a surface integral equation method. Multiple surface plasmon edge modes supported by the fractal structures

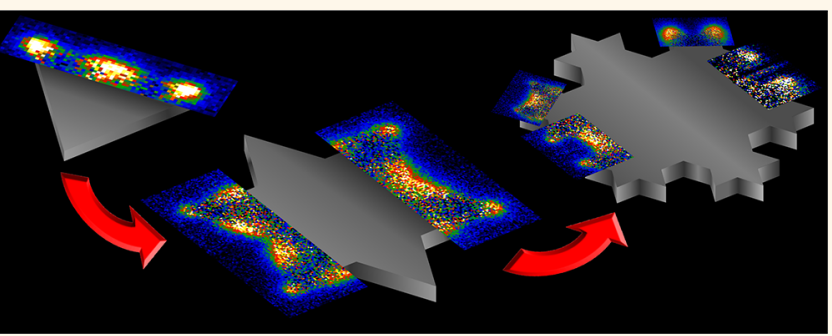
have been imaged and analyzed. Furthermore, by isolating and reproducing self-similar features in long silver strip antennas, the edge modes present in the Koch snowflake fractals are identified. We demonstrate that the fractal response can be obtained by the sum of basic self-similar segments called characteristic edge units. Interestingly, the plasmon edge modes follow a fractal-scaling rule that depends on these selfsimilar segments formed in the structure after a fractal iteration. As the size of a fractal structure is reduced, coupling of the modes in the characteristic edge units becomes relevant, and the symmetry of the fractal affects the formation of hybrid modes. This analysis can be utilized not only to understand the edge modes in other planar structures but also in the design and fabrication of fractal structures for nanophotonic applications.

KEYWORDS: fractals, edge modes, EELS, plasmonics, nanoantennas, Koch snowflake

$\mathrm{T}$ he use of fractal geometries has significantly impacted many areas of science and engineering. One such area is antenna design, where fractal geometries are often utilized in portable communication devices for their compact, broadband characteristics. ${ }^{1,2}$ The term fractal is used to describe curves (most commonly in two dimensions) that present repeating patterns (exact, quasi, or statistical selfsimilarity), at all scale, often obtained by iteratively applying some transformation on a system. ${ }^{3}$ This particular property, as well as their ability to compactly fill space, makes fractals ideal candidates for broadband antennas, and they have indeed inspired the design of several macroscopic antennas that exhibit broadband behavior and improved performance in the $\mathrm{GHz}$ regime. $^{4-9}$

In recent years, interest in a new type of antenna based on surface plasmon resonances, designed to operate at visible light frequencies, has been motivated by potential applications in sensing, ${ }^{10}$ imaging, ${ }^{11}$ energy harvesting, ${ }^{12,13}$ and disease prevention and cure. ${ }^{14}$ These so-called "optical antennas" or "nano-antennas" have characteristic dimensions at nanometerlength scales, requiring nanometer precision for their fabrication and characterization. ${ }^{15}$ With improved nanofabrication tools, including focused ion beam and electron beam lithography (EBL), ${ }^{16}$ their fabrication is becoming increasingly feasible. Early prototype structures studied include dipole $^{17}$ gap, ${ }^{18,19}$ bowtie, $^{20}$ and Yagi-Uda ${ }^{21}$ antennas. The nanoscale dimensions of optical antennas call for demanding characterization requirements and experimental techniques that can image beyond the optical diffraction limit are necessary for the detailed study of subwavelength field confinements in optical antennas. Electron energy loss spectroscopy (EELS), performed in a scanning transmission microscope (STEM), is one of the few techniques which meets all these requirements, combining subnanometer spatial resolution and spectral resolution exceeding $100 \mathrm{meV}^{22}$ Although the EELS energy resolution is lower compared with its optical counterparts, deconvolution techniques and new designs of monochromators can achieve resolutions close to $10 \mathrm{meV} .^{23,24}$ The STEM-EELS technique has been used successfully to map optical excitations in a variety of nanostructure geometries, including triangular prisms, ${ }^{25}$ rods, ${ }^{26,27}$ wires, $^{28}$ cubes, ${ }^{27}$ among others. ${ }^{29-42}$

Received: August 4, 2017

Accepted: October 30, 2017

Published: October 30, 2017 


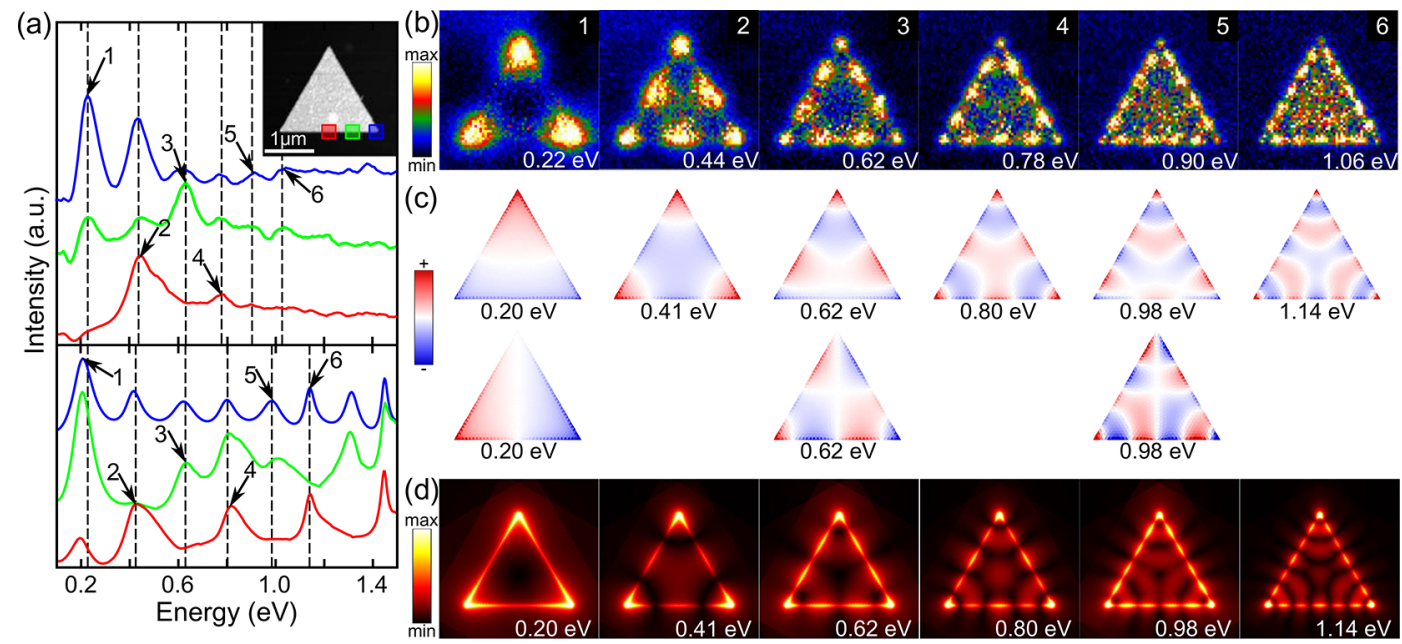

Figure 1. (a) Experimental (top) and computed (bottom) EEL spectra of Koch fractal antenna iteration 0, acquired at the color-coded positions indicated in the ADF image in the inset. The numbers indicate the resonant peaks. (b) Experimental EELS maps of the resonant peaks, indicated in (a), formed by edge modes. (c) Computed charge distributions of the equilateral triangle eigenmodes. The even edge modes only form one eigenmode, while the odd edge modes form two degenerate modes one symmetric and one antisymmetric. (d) Symmetrized near-field intensity distributions of the eigenmodes found in the Koch fractal antennas of iteration 0. Degenerate eigenmodes display identical symmetrized near-field intensities. The EELS maps are obtained at the energies corresponding to the peaks in the EEL spectra $\pm 30 \mathrm{meV}$. The energies indicated in the near-field intensity maps are those of the eigenmodes, not the energy of the peaks in the EEL spectra.

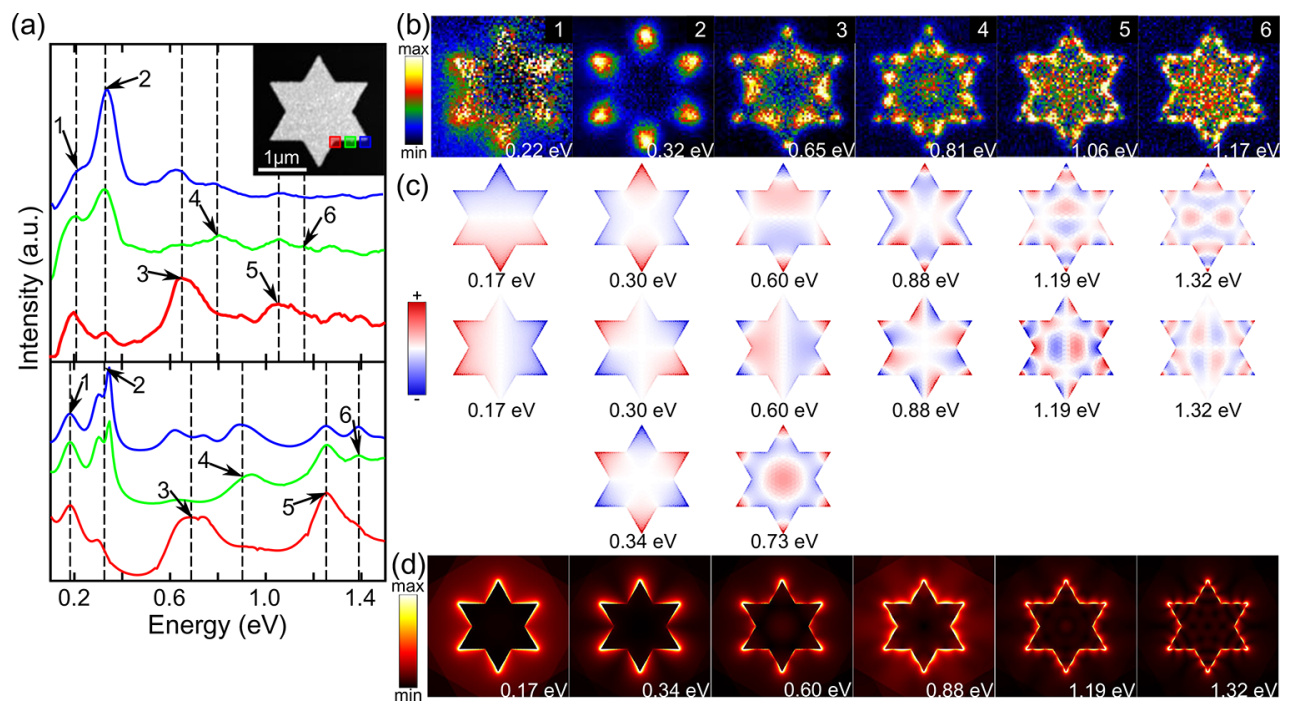

Figure 2. (a) Experimental (top) and computed (bottom) EEL spectra of Koch fractal antenna iteration 1, acquired at the color-coded positions indicated in the ADF image in the inset. The numbers indicate the resonant peaks. (b) Experimental EELS maps of the resonant peaks, indicated in (a), displaying the modes formed by edge modes that can be analyzed by isolating its characteristic " $V$ " edge unit as shown in Figure 3a,b. The analysis shows that the first peak is a dipolar mode, and the next modes are formed by $E_{m}(m=1-5)$ modes in the characteristic edge units. (c) Computed charge distributions of the fractal iteration 1 eigenmodes. All the eigenmodes formed by edge modes are degenerate, and they can be understood considering the interaction of the edge modes within the fractal structure and its symmetry as shown in Figures 4b,c and S9. (d) Symmetrized near-field intensity distributions of the eigenmodes found in the Koch fractal antennas of iteration 1. Degenerate eigenmodes display identical symmetrized near-field intensities.

Early studies of optical fractal antenna designs, including the Cayley tree, ${ }^{43}$ Sierpinski fractals, ${ }^{44-47}$ and other self-similar geometries, ${ }^{48-51}$ suggest that broadband absorption can be achieved in fractal plasmonic nanoantennas. Plasmonic fractal structures have also been tested to improve the efficiency in application such as photovoltaics, ${ }^{52,53}$ extraordinary transmission, ${ }^{47}$ fluorescence enhancement, ${ }^{54}$ third harmonic generation, ${ }^{55}$ and molecular detection. ${ }^{56}$ In this work, we focus on the Koch snowflake fractal geometry. A Koch fractal is constructed by starting with an equilateral triangle (iteration
0 ) and repeating the following procedure iteratively: Divide each edge of the structure into three segments of equal length and then place an equilateral triangle pointing outward in the central segment in each line, that is, the central segment is the base of the new triangle. The fractals are defined by the number of times the described procedure was applied (i.e., an iteration). Figure S1 in the Supporting Information shows the iterations of the Koch fractal geometry. Furthermore, the insets in Figures 1a, 2a, and 5a show annular dark-field (ADF) images of the Koch fractal iterations 0,1 , and 2, respectively. We use EBL to 
fabricate a set of nanoscale fractal antennas, and STEM-EELS is used to image the optical excitations supported by the structures (see Methods section). The high spatial resolution achieved with STEM-EELS allows us to visualize the multiple plasmonic modes supported by the fractal structures, to analyze the structural origin of the modes present, and to study the effect of self-similarity by comparing the response of different fractal geometry iterations. The experimental results are complemented with numerical calculations of both EELS spectra and eigenmodes, as described in the Methods section. In this work, we show that the localized plasmon resonances in a complex geometry such as the Koch snowflake fractal follow simple scaling rules based on the number of self-similar segments found in the structure. These scaling rules may be used in the design of fractal antennas for applications in sensing and compact nanophotonic architectures.

\section{RESULTS AND DISCUSSION}

To analyze the effect of self-similarity on the spectral response of metallic nanoantennas, silver Koch snowflake fractal antennas of iterations 0,1 , and 2 have been fabricated, as shown in the insets in Figures 1a, 2a, and 5a. The iteration 0 structure has a side length of $2 \mu \mathrm{m}$ and a thickness of $30 \mathrm{~nm}$. The first and second iteration structures maintain a thickness of $30 \mathrm{~nm}$. Figures 1a, 2a, and 5a show the experimental (top) and simulated (bottom) EELS spectra of the fractal structures obtained at several positions indicated by the color-coded boxes in the insets. Overall, we observe a fair agreement between the simulation and the experiment with plasmon peaks and energies well reproduced. The increasing difference of the peaks energy between experiment and simulation is assumed to be due to the absence of the substrate in the simulations, whose influence can change with increasing mode energy, ${ }^{57}$ as well as possible deviations of the actual Ag permittivity from the Drude model used for computations. The spectral response of each structure exhibits several surface plasmon resonances with a first resonant peak at approximately the same energy $(0.22 \pm$ $0.04 \mathrm{eV}$ ) for all the iterations of the Koch fractal behavior. The simulations confirm this effect.

Edge Modes and Koch Fractal Iteration Zero. As previously described in the literature, the resonances in a planar structure can be described as quasi one-dimensional resonances (edge modes) along the edge of a structure. ${ }^{33,58-60}$ Even the fundamental dipolar and quadrupolar modes can be described in terms of edge modes. ${ }^{61}$ An edge mode of order $m$ is noted $E_{m}, m$ being the number of nodes along the edge. Also, edge modes can be designated as odd or even depending on the number of nodes $m$ (or, equivalently, with opposite or identical charges at the edge extremities). The maps in Figure $1 \mathrm{~b}$ show the resonances of the fractal iteration 0 that display along each one of the edges the characteristic node distribution of edge modes. Thus, the first resonant peak at $0.22 \pm 0.03 \mathrm{eV}$ corresponds to a dipolar mode or edge mode order one $\left(E_{1}\right)$, and the following peaks can be identified as formed by edge modes order two $\left(E_{2}\right)$, three $\left(E_{3}\right)$, four $\left(E_{4}\right)$, five $\left(E_{5}\right)$, and six $\left(E_{6}\right)$ at $0.44,0.62,0.78,0.90$, and $1.06 \mathrm{eV}$ respectively (with an effective energy resolution of $30 \mathrm{meV}$ ). These results are supported by simulations of the eigenmodes, in Figure $1 \mathrm{c}$, that show the charge distribution of edge modes at the edges of the equilateral triangle according to the symmetry of the nanostructure.

Based on the symmetry of the Koch fractal structures, we will designate as symmetric or antisymmetric the eigenmodes with symmetric or antisymmetric charge distributions with respect to the vertical axis. Due to the structure symmetry, each eigenmode having only the mirror symmetry (symmetric or antisymmetric) will implicitly exist 3 times for the iteration 0 and 6 times for higher iterations. Since this does not bring any additional information, this "degeneracy" will not be mentioned further, and the term degeneracy will only refer to eigenmodes having the same energy but different charge distributions that cannot be matched by mirror or rotation operation. The choice of the vertical axis as the reference axis is arbitrary, and considering one of the other two symmetry axes would give the same results. EELS maps also follow the structure symmetry because the signal is obtained by exciting and probing at the same location, the simplest example being the resonances of a nanodisk that appear like concentric rings. ${ }^{62}$ For the same reason, Koch snowflake fractals EELS maps always have a $C_{3}$ or $C_{6}$ symmetry, for iteration 0 and higher, respectively. Otherwise, this is not the case for all the computed eigenmodes, and to be able to identify them easily in the EELS maps, the electric near-field intensity of each eigenmode (Figures 1d, 2d, and $5 \mathrm{~d}$ ) is "symmetrized" by adding itself 3 times following $0^{\circ}$, $120^{\circ}$, and $240^{\circ}$ rotations. These symmetrized maps provide a qualitative link between the measurements and the simulations. Interestingly, we observe that degenerate eigenmodes give the same symmetrized near-field map, despite different charge distributions. The electric near-field intensities of the eigenmodes before being symmetrized are shown in in Figures S2-S4.

Following this description, the eigenmodes present in the equilateral triangle (Koch fractal of iteration 0 ) can be analyzed. Figure $1 \mathrm{c}, \mathrm{d}$ shows the surface charge distributions and the simulated, symmetrized, electric near-field intensity distributions of the eigenmodes for the fractal iteration 0 . From the surface charge distributions, it is clear that all three edges of the triangle in the eigenmodes of the resonant peaks 2,4 , and 6 display a charge distribution corresponding to a one-dimensional mode of the same order $E_{2}, E_{4}$, and $E_{6}$ (with even edge modes), respectively. For the case of the eigenmodes formed by $E_{1}, E_{3}$, and $E_{5}$ (with odd edge modes), two degenerate eigenmodes are present: one in which two edges have the same charge distribution corresponding to an edge mode (symmetric eigenmode), and another eigenmode in which only one edge exhibits the charge distribution corresponding to an edge mode and the two other edges have opposite charge distribution sign relatively to each other (antisymmetric eigenmode). The eigenmodes $E_{2}, E_{4}$, and $E_{6}$ follow the same $C_{3}$ symmetry as the triangle, whereas other eigenmodes only have the mirror symmetry with degeneracy two (antisymmetric and symmetric respectively to the vertical axis). Based on the symmetry of the triangle, the formation of two degenerate eigenmodes for odd edge modes is understandable. This is due to the fact that in all odd edge modes the charge at the extremities of the edge (the triangle corner) must be opposite, and in a triangle only two edges at maximum can fulfill this constraint at the same time. The odd edge modes therefore split into symmetric and antisymmetric degenerate edge modes. The surface charge distributions of the eigenmodes of iteration 0 confirm that it is possible to describe them as formed by edge modes.

Characteristic Edge Units and Koch Fractal Iteration one. As is the case of the Koch fractal iteration 0 , the lowest energy mode in the fractals of iteration 1 is also identified as a dipolar mode. Although the geometry modification from iteration 0 to 1 is large, the energy of the dipolar mode shifts 
only by $30 \mathrm{meV}$ in the simulations. Figure $2 \mathrm{c}$ shows the surface charge distributions for the modes of the fractal iteration 1. For the dipolar mode, two degenerate eigenmodes are supported, one with a vertical dipole moment and the other with a horizontal dipole moment, as observed for the dipolar modes of the fractal iteration 0 . However, the higher order modes of the Koch snowflakes of iteration 1 display complex EELS maps and charge distributions as observed in Figure $2 b, c$. To understand these complex modes, the edges of the fractal structure are divided into characteristic edge units, composed of two segments with a $120^{\circ}$ angle between them ("V" shape), as shown in Figure S1 and in the inset of Figure 3a. To isolate the characteristic edge unit of the Koch fractal iteration 1, we reproduced this "V" shape at the end of a $50 \mu \mathrm{m}$-long silver strip (extending vertically beyond the insets in Figure 3 ). Figure 3a,b shows the EELS spectra and energy-filtered maps of the silver strip representing the characteristic edge units of the
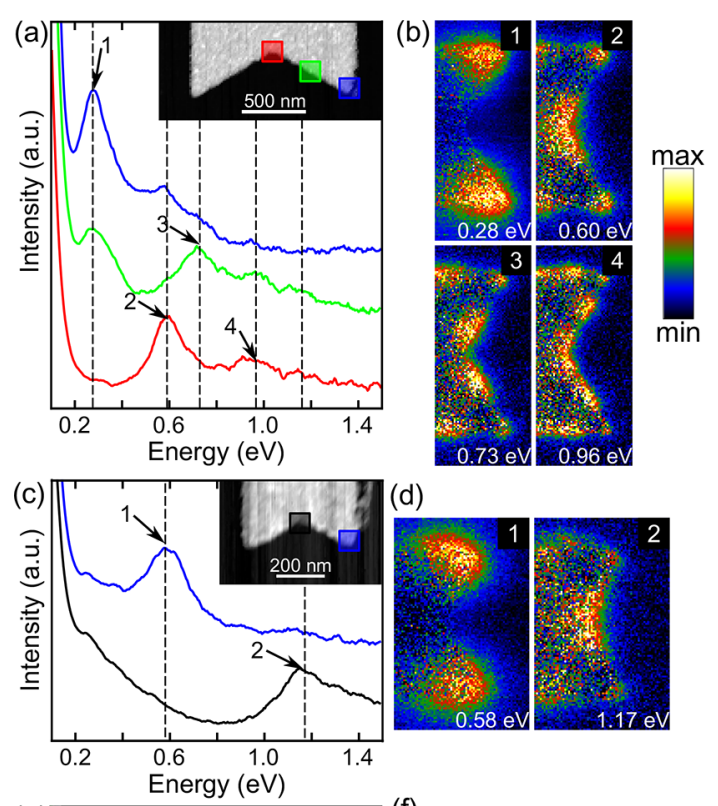

(d)
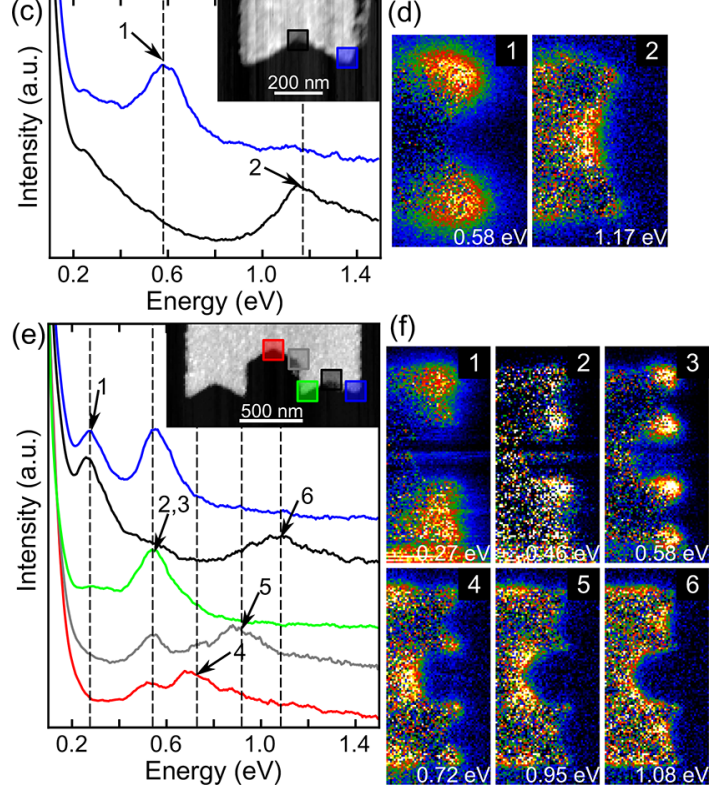

Figure 3. Experimental EEL spectra of the characteristic edge units of Koch fractal antennas of iterations 1 (a) and 2 (c,e) reproduced at the very end of a $50 \mu \mathrm{m}$ long silver strip, acquired at the colorcoded positions indicated in the ADF image in the insets. The arrows indicate the peaks corresponding to resonant edge modes. EELS maps of the plasmon modes found in the characteristic edge units of Koch fractal antennas of iteration 1 (b) and 2 (d,f). The second peak in (e) is labeled as " 2,3 " because it represents two different resonant modes that are energetically close. The modes have been separated with a Gaussian fit yielding EELS maps 2 and 3 , in (f), at $0.46 \pm 0.07 \mathrm{eV}$ and $0.58 \pm 0.09 \mathrm{eV}$, respectively.
Koch snowflake fractal iteration 1 . Here we are able to identify four resonant peaks that correspond to edge modes, in a similar manner to the modes found in straight edges on silver strips, ${ }^{58,60}$ despite the fact that the characteristic edge unit of the Koch fractal is formed by two edges at an angle. Hence, the EELS intensity distribution of these resonant peaks corresponds to edge modes of order one $\left(E_{1}\right)$, two $\left(E_{2}\right)$, three $\left(E_{3}\right)$, and four $\left(E_{4}\right)$. A similar behavior was found in bent silver nanowires in which the plasmon modes were unaffected by the presence of kinks. ${ }^{28}$

To further support the hypothesis that bent edges will maintain the same quasi one-dimensional modes than those found in straight edges, let us draw a parallel with the onedimensional modes of silver nanowires. We performed simulations of a straight wire and two bent wires at $120^{\circ}$ and $60^{\circ}$ as shown in Figure S5. For a $120^{\circ}$ bending, which is the angle of the characteristic unit in the Koch fractals, the bending produces only an energy shift of the odd edge modes, compared to the straight wire, while maintaining their nodal distributions. For a $60^{\circ}$ bending, modes $E_{3}$ and $E_{4}$ begin to merge. The angle at which this process occurs is characteristic of each pair of modes and depends on the order of the modes and the dimensions of the wires. A detailed study of this interaction is beyond the scope of this work and will be discussed elsewhere.

Based on the observation that bent edges can support edge modes equivalent to those in straight edges, we can now compare the EELS maps of the isolated edges that form a characteristic edge unit with those of the Koch snowflake iteration 1. From the EELS energy filtered maps shown in Figures $2 b$ and $3 b$, we can recognize that the second peak in the snowflake, at $0.32 \mathrm{eV}$, is formed by edge modes of order one $\left(E_{1}\right)$ or edge dipolar modes in each one of its characteristic edge units. In a similar manner, the third, fourth, fifth, and sixth peaks in Figure $2 \mathrm{a}$ are formed by edge modes of order two $\left(E_{2}\right)$, three $\left(E_{3}\right)$, four $\left(E_{4}\right)$, and five $\left(E_{5}\right)$, respectively, by comparison with the isolated characteristic "V" edge unit. The excellent agreement between the nodal distributions observed in the EELS maps of the isolated edge units (strip extremities) and the snowflake iteration 1 is revealed by a side-by-side comparison, shown in Figure S6. If we compare the spectra from the Koch snowflake and the isolated characteristic "V" edge unit in Figures 2a and 3a, respectively, we observe that the peak corresponding to the $E_{1}$ edge mode in the isolated edges is red-shifted by $70 \mathrm{meV}$ with respect to the second mode in the snowflake. If we align these peaks by red shifting the spectra in the Koch snowflake iteration 1 , as shown in the side-by-side comparison in Figure S6a, then we observe an excellent match between the peaks present in the Koch snowflake fractal and the modes of the isolated edge unit in the $50 \mu \mathrm{m}$ silver strips. This supports the analysis of the charge and nodal distribution of the modes in the fractal and confirms that the modes in the Koch fractal are equivalent to the modes in the isolated edge units. The small energy difference between the modes present in both structures can be attributed, to a greater extent, to the interaction between the edge modes within the Koch snowflake $^{61}$ and to imperfections in the fabricated structures.

Eigenmodes and Coupling of Edge Modes. In the simulated EELS spectra of the Koch snowflake iteration 1 (Figure $2 \mathrm{a}$ ), we notice that the second peak at $0.32 \mathrm{eV}$, which is formed by $E_{1}$ modes, is actually composed of two close resonant peaks separated by only $46 \mathrm{meV}$. Due to the intrinsic and experimental broadening, the experimental EEL spectra 
cannot resolve those two peaks, and thus only one peak appears. To further analyze the formation of these two peaks, we simulated the EELS spectra of Koch snowflake fractals iteration 1 of several sizes while maintaining the thickness of the structure constant as shown in Figure 4a. The changes in
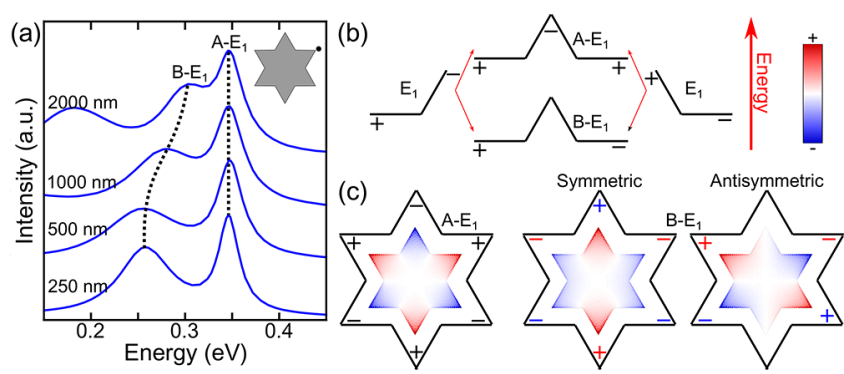

Figure 4. (a) Simulated spectra of Koch fractal iteration 1 for several initial side lengths of the fractal. The spectra are shifted to align the second resonant peak. The two peaks show the evolution of the interaction of the bonding (B) and antibonding (A) $E_{1}$ edge modes; as the structure shrinks, a stronger interaction between $E_{1}$ modes results in larger splitting energy of these peaks. The inset shows the position where the spectra were calculated. (b) Energy diagram (not to scale) showing the formation of bonding and antibonding modes due to the coupling of two $E_{1}$ edge modes. (c) Charge distribution diagrams of the bonding and antibonding $E_{1}$ modes in Koch fractal iteration 1. Due to the symmetry of the structure, the bonding mode supports two degenerate eigenmodes; the calculated eigenmodes are displayed inside the diagrams.

these two peaks, as we change the size of the fractal, will indicate if these two peaks are produced by the coupling of edge modes. As the size of the fractal reduces, all of the peaks will blue-shift. However, we want to examine the energy splitting between the modes formed by $E_{1}$ edge modes, and not the shift in energy due to the different fractal sizes; therefore, we align the second peak of each fractal to the second peak in the fractal with the initial length of $2 \mu \mathrm{m}$. To this end, the spectra of the fractals of initial length of $1 \mu \mathrm{m}, 500 \mathrm{~nm}$, and 250 $\mathrm{nm}$ were red-shifted by $0.27,0.69$, and $1.2 \mathrm{eV}$, respectively. The energy splitting is a measure of coupling strength, and we observe that, as we increase the size of the fractal, the energy splitting reduces. This behavior corresponds to a mode splitting caused by coupling of edge modes within a structure, as shown in the work of Schmidt et al. in rectangular structures. ${ }^{61}$ Thus, this might suggest that interaction of the $E_{1}$ edge modes is responsible for the formation of these two peaks. As the size of the fractal increases, the distance between edges increases, and the interaction between edge modes is weaker, thus the energy splitting is reduced.

The simulations also show that the second and third resonant peaks, observed experimentally, are formed by three eigenmodes each, two eigenmodes being degenerate as shown in Figure 2c. Although the experimental results could be explained by neglecting the formation of these three eigenmodes, their charge distributions indicate the interaction of edge modes within the fractal structure. To understand the charge distributions of the eigenmodes in the Koch fractal iteration 1 , we use the hybridization model. ${ }^{63}$ When edge modes interact, the hybridization model dictates the formation of a bonding and an antibonding mode. In the case of the Koch fractal formed by connected characteristic edge units, the hybridized modes become bonding (B) and antibonding (A) charge-transfer hybridized modes, ${ }^{64,65}$ as shown in Figures $4 \mathrm{~b}$ and $S 9$ for modes $E_{1}$ and $E_{2}$, respectively. These modes can be understood intuitively as the coupling of two edges through a conductive junction (using the terminology from ref 65) in which charges can indeed be "transferred". From Figure $4 b$, we observe that the $E_{1}$ antibonding edge mode is formed by two $E_{1}$ modes with equal charges at the junction which, due to the charge-transfer, merge and become one antinode. The $E_{1}$ bonding mode is formed by opposite charges at the junction that cancel each other by charge transfer, leaving the bonding modes with two opposite charges at the extremities. The $C_{3}$ symmetry of the Koch fractal would allow a configuration in which all the edges can display the antibonding modes as shown in Figures $4 \mathrm{c}$ and $S 9$ for edge modes $E_{1}$ and $E_{2}$, respectively. However, for the bonding modes, in which the charges at the extremities are opposite, the symmetry only

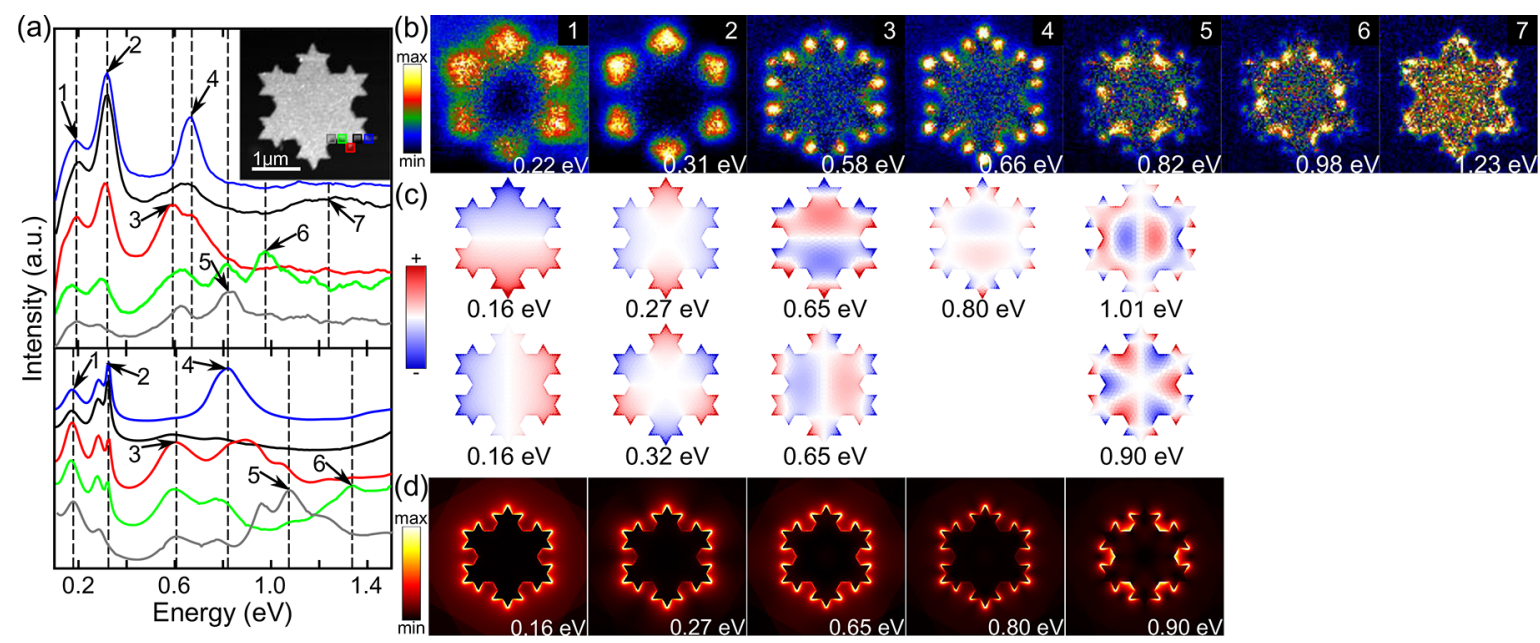

Figure 5. (a) EEL spectra of Koch fractal antennas of iteration 2 (experimental (top) and computed (bottom)) acquired at the color-coded positions indicated in the ADF image in the inset. The arrows indicate the peaks corresponding to resonant modes. (b) Experimental EELS maps of the resonant peaks, (c) computed charge distributions, and (d) near-field intensity distributions of the eigenmodes found in the Koch fractal antennas of iteration 2. The EELS maps are obtained at the energy corresponding to the peak in the EEL spectra $\pm 30 \mathrm{meV}$. The energy indicated in the near-field intensity map is the one of the eigenmode, not the energy of the peak in the EEL spectra. 
allows two sides of the structure to fulfill this constrain. As it was the case for the edge modes in Koch fractal iteration 0, this constraint promotes the formation of a symmetric and an antisymmetric degenerate eigenmodes. The symmetric eigenmodes can be classified into two distinct groups, each group formed by bonding edge modes at the left and right sides of the fractal (blue and red \pm signs in Figures $4 \mathrm{c}$ and S9): one group with a junction between bonding edge modes at the top apex and the other with the junction at the bottom apex. For the antisymmetric eigenmodes, two bonding edge modes at opposite upper and lower part of the fractal are observed (blue and red \pm signs in Figures $4 \mathrm{c}$ and S9), displaying indeed an antisymmetric charge distribution. This description of the formation of eigenmodes in the Koch fractal order 1 based on the hybridization of edge modes suggests that the eigenmodes are formed by the interaction of edge modes within the fractal structures. However, an in-depth analysis of edge modes coupling that goes beyond the purpose of this work is required to confirm this hypothesis. In this analysis, we show how the edge modes formed in the characteristic "V" edge units and the symmetry of the structure determine the three different charge distributions of eigenmodes. Equivalently, the energy loss peaks four, five, and six in Figure 2a that are formed by edge modes of order three, four, and five, respectively, exhibit eigenmodes. However, for these peaks, we were only able to find two degenerate eigenmodes as shown in Figure 2c. Indeed, the symmetry and spectral proximity between the expected additional nondegenerated mode and the degenerated ones make their numerical extraction very difficult.

Koch Fractal Iteration 2. The edge isolation approach is now applied to understand the structure modes present in the Koch fractal of iteration 2. We therefore divided the edges of the snowflake structure to find the characteristic edge unit, which is the same "V" shape as for the iteration 1 but one-third smaller, as shown in Figures $3 \mathrm{~d}$ and S1. In the EELS spectra and energy filtered maps of the isolated characteristic shape, shown in Figure 3c,d, two resonant peaks that correspond to edge modes $E_{1}$ and $E_{2}$, similar to those found in the iteration 1 fractal, but at higher energies due to the shorter length of the edge, are identified. A comparison of these two modes, excited in the characteristic isolated edge unit, with the modes present in the Koch snowflake fractal of Figure 5a,b enables us to identify peak four of the fractal as formed by edge mode order one $\left(E_{1}\right)$ of the isolated edge (because of the same strong EELS signal on each small vertex) and peak seven of the fractal as formed by an edge mode order two $\left(E_{2}\right)$ of the isolated edge on the strip. To corroborate this argument, a side-by-side comparison of the maps and spectra of the isolated characteristic "V" edge unit and the snowflake fractal iteration 2 is shown in Figure S7. This comparison emphasizes the excellent nodal distribution agreement of the modes between the Koch fractal and the isolated edge. As we did in the comparison between the fractal iteration 1 and its characteristic isolated edge unit, for iteration 2 we also red shift the spectra in the Koch snowflake iteration 2 by $70 \mathrm{meV}$, as shown in Figure S7a. We observe that, after this shift, the energy peaks corresponding to the $E_{1}$ and $E_{2}$ modes in the isolated characteristic units in the $50 \mu \mathrm{m}$ silver strips match the peaks present in the Koch snowflake fractal.

For the fractal structure iteration 2, we also isolate and analyze a larger portion of the structure, as shown in Figure $3 e, f$, that is, the characteristic edge unit of the Koch fractal iteration 1 to which an additional fractal iteration is applied. In this larger portion of the fractal, five peaks are observed, as shown in Figure 3e. The first peak at $0.27 \pm 0.04 \mathrm{eV}$ corresponds to a $E_{1}$ mode or dipolar mode, which is the same mode displayed in the silver strip of the Koch fractal iteration 1 at $0.28 \pm 0.04 \mathrm{eV}$. This result suggests that the dipolar mode of iteration 1 can still be excited in the next iteration of the fractal as the dipolar mode is the most fundamental one (as seen in Figure 5c) and is thus expected to be marginally affected by the small modification of the second iteration. This effect is also observed in the Koch snowflake fractal iteration 1 (Figure 2a), where the second peak, which is a dipolar edge mode $\left(E_{1}\right)$, at $0.32 \pm 0.04 \mathrm{eV}$ is also present in the Koch snowflake iteration 2 (Figure $5 \mathrm{a})$ at $0.31 \pm 0.04 \mathrm{eV}$.

The second peak in Figure $3 e$ is labeled as " 2,3 " because in the spectrum image, two dissimilar intensity localizations, one with the antinodes located in the inner vertices and the other located in all vertices, are observed in the maps two and three in Figure 3f, respectively. To extract the EELS maps corresponding to each surface plasmon resonance, we isolate the contribution from each edge mode to the spectrum image using the nonlinear least-squares fitting tool of the "Digital Micrograph" software, ${ }^{66}$ which fits Gaussian peaks to a spectrum image. The separation fits two Gaussians to the peak at $0.55 \mathrm{eV}$ yielding the two EELS maps at $0.46 \pm 0.07 \mathrm{eV}$ and $0.58 \pm 0.09 \mathrm{eV}$ for the second and third modes, respectively. The third and sixth peaks of the isolated characteristic edge units of fractal iteration 2 on the strip shown in Figure $3 \mathrm{e}, \mathrm{f}$ are the same two modes found in the isolated characteristic edge unit of Koch fractal iteration 2 shown in Figure 3c,d. Thus, the modes of the isolated characteristic edge units can be described as edge modes order one $\left(E_{1}\right)$ and two $\left(E_{2}\right)$ and represent peaks four and seven found in the Koch snowflake fractal iteration 2 in Figure 5. These results confirm that the modes present in the strips are a good representation of modes present in the Koch snowflakes.

When two of the characteristic "V" edge units of Koch fractal iteration 2 are joined by a $120^{\circ}$ angle, an inverted " $U$ "-like shape is generated as shown in the central area of the inset in Figure $3 \mathrm{e}$ and in Figure S1. This characteristic "U" shape, despite the multiple kinks, also sustains edge modes. Modes $E_{1}$ (dipolar edge mode), $E_{2}$, and $E_{3}$ are identified at $0.46 \pm 0.07$, $0.72 \pm 0.04 \mathrm{eV}$, and $0.95 \pm 0.04 \mathrm{eV}$ as seen in Figure 3f. This "U" shape is also present in the full Koch snowflake fractal iteration 2 in Figure 5, and by comparison, it displays the same edge modes found in the isolated edge seen in Figure 3e,f. The third, fifth, and sixth peaks in the full Koch snowflake can be identified as being formed by $E_{1}$ or dipolar edge mode, $E_{2}$, and $E_{3}$ edge modes, respectively. To confirm this identification, Figure S8 shows the good match of the nodal distributions between the modes present in the Koch snowflake fractal iteration 2 and the modes isolated in the $50 \mu \mathrm{m}$ silver strips. The spectra in Figure S8 also shows a good energy overlap between the modes in the isolated characteristic edge units on the strip and the modes of the snowflakes after the latter is redshifted by $70 \mathrm{meV}$. This nodal and energy match supports the evidence that the isolated modes on the silver strip are equivalent to the modes present in the Koch snowflake fractals. The energy shift can be mainly attributed to interaction of edge modes in the snowflake ${ }^{61}$ and to a lower extent to the fabrication procedure that did not produce equal edges and sizes in both structures and locally modifies the plasmon response. ${ }^{67}$ To support the proposed concept that a "U" characteristic unit structure can sustain plasmonic edge modes similar to the ones found in a straight edge, we draw a parallel 
and analyze the modes in a simulated " $U$ " shape bent silver nanowire. Figure S10 shows the spectra and the energy-filtered maps of the bent nanowire, demonstrating that, despite the bending, the nanowire still supports edge modes. This was also demonstrated by Rodríguez-Fortuño et al. ${ }^{68}$

Self-Similarity of Edge Modes. Now that the modes present in the Koch snowflake fractals have been identified, we can analyze their self-similarity (i.e., fractal character) as the number of iterations increases. Due to the $C_{3}$ symmetry of the Koch snowflake fractal, each mode exists 3 times. As stated above, we ignore this "degeneracy" in our analysis. For each edge mode in the Koch snowflake fractal structure, multiple eigenmodes can be found. However, in our analysis of selfsimilarity of edge modes, we will not consider these eigenmodes, and we only focus on the modes found experimentally. Figure 6 shows a diagram depicting the

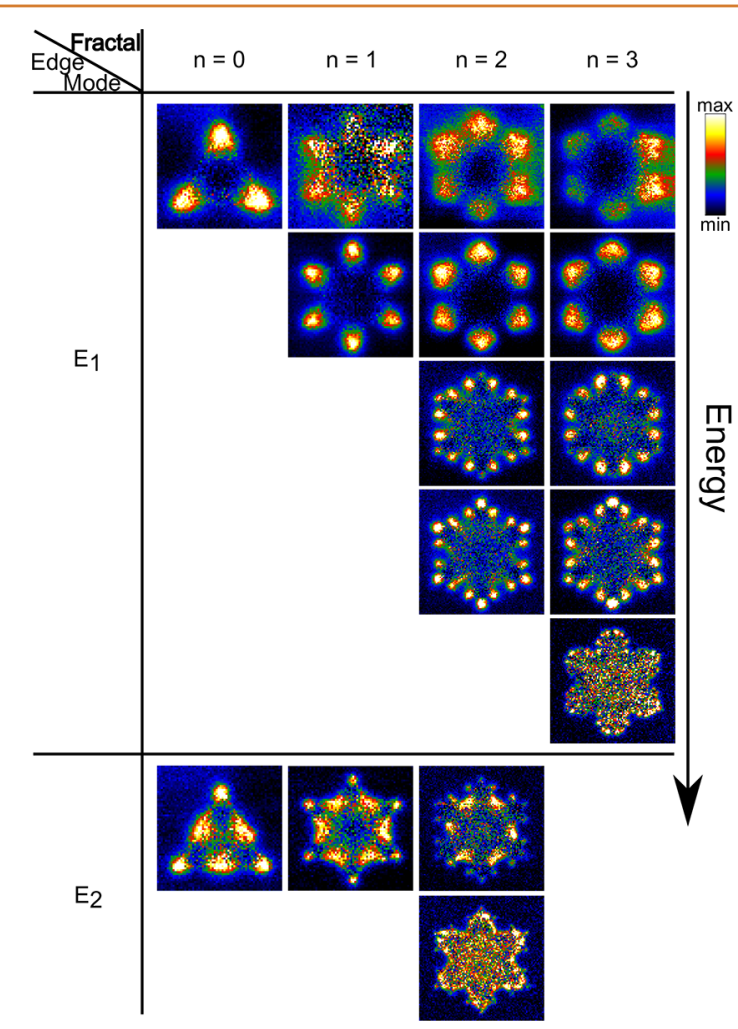

Figure 6. Diagram of the formation of the plasmon modes in Koch snowflake fractal antennas. The diagram shows the fractal character of the Koch snowflake structures, in which the dipolar $/ E_{1}$ modes of each iteration are carried to higher iterations. Due to the small size of the features in iteration 3 , the characteristic edge units were not well reproduced, and only a few edges supported $E_{1}$ modes as seen in the EELS map.

evolution of edge modes as the Koch fractal iteration increases. For the iteration 0 , only one mode of a particular order is observed for each edge. In the Koch snowflake fractal of iteration 1 , two modes that originate from a dipolar/ $E_{1}$ mode and only one mode formed by each higher order edge mode are observed. The first mode is the same dipolar mode found in iteration 0 , and the second mode is formed by the $E_{1}$ modes in the characteristic "V" edge unit in this iteration as shown in Figures $3 \mathrm{c}$ and $\mathrm{S} 1$. In the case of the Koch fractal iteration 2, four modes formed by dipolar $/ E_{1}$ edge modes are present, one coming from fractal iteration 0 , one from the $E_{1}$ mode of fractal iteration 1 , and two new modes originated by $E_{1}$ modes. As discussed previously, this structure presents two types of characteristic edge units, one is the characteristic "V" shape and the other is the " $U$ " shape as shown in Figures $3 \mathrm{e}$ and $S 1$, and each one of these two units can support edge modes, thus two new $E_{1}$ formed modes are created during this iteration. Also two new modes, one per type of characteristic edge unit, of a particular higher order are supported. Fractals of iterations 0 and 1 have only one type of characteristic edge unit, therefore, only one mode is supported for each higher order edge mode. From this analysis, the fractal character of the Koch snowflake is reflected in the number of dipolar $/ E_{1}$ modes supported by the fractal structure. In addition, the total number of edge modes in a Koch snowflake is equal to the number of dipolar/ $E_{1}$ modes in the previous fractal iteration plus the modes formed on all the characteristic edge units present in the fractal. In Figure 6, we also observe Koch iteration 3 that we have not analyzed in the previous sections. The small features introduced in iteration 3 make the fabrication as well as the simulation challenging. However, as seen in the $\mathrm{ADF}$ in Figure S11, we were able to fabricate and reproduce some of the features of iteration 3. The EELS maps of iteration 3 show that the fractal character of the Koch snowflake fractal is also identified in this iteration, with the dipolar $/ E_{1}$ modes of the previous iterations also supported in this iteration. Due to the fabrication constrains, the characteristic edge units of iteration 3 are not well reproduced, but despite this fact, we can observe the antinodes corresponding to $E_{1}$ modes in a few of the smaller corners of the fractal. Because the experimental results show that the modes in the fractal are governed by the modes in the characteristic edge units, this analysis can be applied to other planar fractal structures.

Now that we understand how the modes evolve as the fractal iteration increases, we can extend and quantify the number of dipolar/ $E_{1}$ modes $\left(N_{n}^{\text {modes }}\right)$ generated by the self-similarity in the Koch snowflake fractal iteration " $n$ ". From this analysis, one can infer how many types of characteristic edge units in the Koch fractal are produced after " $n$ " iterations of the fractal. For $n=0$ and 1 , only one type of characteristic edge unit is produced, however for $n>1$, after each iteration two types of characteristic edge units are produced, the " $V$ " and the "U" shapes. Therefore, $N_{0}^{\text {modes }}=1$, and for $n>0, N_{n}^{\text {modes }}=2 n$. Additionally, the Koch fractal structure will have one mode for each higher order mode for $n=0,1$ and two modes for each higher order mode for $n>1$. The results show that the plasmons excited on Koch snowflake fractal structures show a self-similar fractal response with the number of modes increasing after each iteration. This confirms that plasmonic fractal optical antennas can exhibit a multiresonant or broadband behavior while maintaining a compact structure similar to those found in macro-scale antennas. The simple scaling rule described here can be used for nanoantenna design. However, this scaling has limits due to mode coupling within the fractal, as pointed out in the coupling of edge modes section. The smaller the structure, the stronger is the effect of the coupling. In measurements where coupling within the fractal is relevant, the symmetry of the fractal structure plays a critical role, as shown above. In that case, one can also think of the fractal structure as the sum of characteristic edge units, each one contributing the basic components for the formation of the fractal eigenmodes. Taking into consideration the coupling of edge modes ${ }^{60}$ and analyzing how the symmetry affects the nodal distribution and therefore the hybridization of the modes, the final distribution of the hybrid modes could be predicted. 
This analysis can be applied to other 2D fractal structures to understand how the fractal character affects the plasmon modes and guide the design and fabrication of fractal structures for nanophotonic applications.

\section{CONCLUSION}

The plasmon modes present in planar silver Koch snowflake fractal antennas have been investigated using EELS and numerical computations. The lowest energy modes present in all of the fractal structures were identified as dipolar modes. For the higher energy modes, insight into their origin was gained by measuring simplified geometries describing the basic building block segments of the fractal structures. Two types of basic segments were found and studied: a "V" characteristic edge unit, formed by two lines at $120^{\circ}$ angle, and a "U" characteristic edge unit, formed joining two characteristic " $V$ " edge units also at $120^{\circ}$ angle. In spite of the fact that these two geometries presented were not straight-line segments, the modes sustained were edge modes $\left(E_{i}\right)$. All the edge modes supported in the Koch snowflake fractal have been identified from an analysis of the isolated characteristic edge units. Interestingly, the fractal response can be obtained by the sum of the modes supported by its characteristic edge units that are the basic components of the fractal. We also showed that, as the size of the fractal structure is reduced, the coupling within the structure can become relevant and how the influence of the symmetry of the fractal on edge mode coupling of the basic components must be considered. When the coupling is weak and it can be neglected, the total number of edge modes in a Koch snowflake of a given number of iterations depends on the number of characteristic edge units created in the fractal. Introducing this simple rule, we observed that the number of plasmon dipolar $/ E_{1}$ edge modes increases by two after a fractal iteration, confirming that the plasmonic Koch snowflake fractal antennas can exhibit a multiresonant or broadband behavior while maintaining a compact structure reflecting the characteristics of their macroscale counterparts.

\section{METHODS}

Simulations. The computation of the EELS spectra and the eigenmodes of the Koch snowflake fractals were done using a full wave surface integral equation (SIE) method. ${ }^{69,70}$ The fractal structures are considered in a homogeneous medium with a permittivity of $\epsilon=1.8$ to account for the substrate influence. A Drude model was used for the permittivity of Ag with a plasma frequency $\omega_{p}=9.3 \mathrm{eV}$, losses $\gamma=0.03$ $\mathrm{eV}$, and $\epsilon_{\infty}=4.3$. The simulations of the bent nanowires were performed using the MNPBEM toolbox ${ }^{71,72}$ that uses the boundary element method to solve Maxwell's equations. The wires were calculated using a tabulated dielectric function, ${ }^{73}$ and a permittivity of 2 for the medium surrounding the structure to account for the substrate influence. A comparison between SIE and BEM can be found in the Supporting Information of ref 69.

Sample Fabrication. The structures were fabricated by EBL directly onto silicon nitride TEM grids. ${ }^{23}$ We deposited poly(methyl methacrylate), 950,000 molecular weight at $3 \%$ anisole, on a $50 \mathrm{~nm}$ thick silicon nitride TEM grid (Norcada) by spin coating at $6000 \mathrm{rpm}$. The sample was then baked at $175{ }^{\circ} \mathrm{C}$ for $2 \mathrm{~min}$. The patterning was performed on a JSM-7000F SEM (JEOL) equipped with Nano Pattern Generation System (NPGS). Development was done in a 3:1 isopropyl alcohol:methyl isobutyl ketone solution for $70 \mathrm{~s}$ and rinsed in isopropyl alcohol and dried with $\mathrm{N}_{2}$. Ag was deposited in an electron beam evaporator system, with a $6 \mathrm{keV}$ and $250 \mu \mathrm{A}$ electron source. Finally, the lift-off was done by soaking the sample in acetone, followed by isopropyl alcohol rinsing.
EELS Measurements. The EELS spectral response of the structures was characterized using an ultrastable STEM-TEM (FEI Titan 80-300) equipped with an electron monochromator. We focused an $80 \mathrm{keV}$ electron beam on the sample and rastered it over a region of interest, and we simultaneously acquired signals from an annular darkfield detector and an EELS spectrometer (Gatan Imaging Filter, Tridiem model 865) from each position in the raster scan. The spectra were recorded with an exposure time of $1 \mathrm{~ms} /$ spectrum and a dispersion of $10 \mathrm{meV}$ per channel for the Koch snowflake fractals and $5 \mathrm{meV}$ per channel for the isolated characteristic edge units. To further improve the energy resolution, we performed the Richarson-Lucy algorithm procedure, ${ }^{23}$ achieving an effective energy resolution up to $30 \mathrm{meV}$ in our spectrum images. Each spectrum image was deconvoluted using seven deconvolution iterations and normalized by the total electron count at each pixel position. To have a better comparison between experiments and simulations, that do not have the "zero-loss-peak" (ZLP), we subtract it from the spectra. After deconvolution, we remove the ZLP contribution to the spectra by fitting the ZLP tails using a power function.

\section{ASSOCIATED CONTENT}

\section{Supporting Information}

The Supporting Information is available free of charge on the ACS Publications website at DOI: 10.1021/acsnano.7b05554.

Calculated eigenmodes of three iterations of the Koch snowflake fractal and their near-field intensity distribution. Diagram depicting the formation of eigenmodes by coupling of $E_{2}$ modes in Koch snowflake iteration 1 along with their calculated eigenmodes. Side-by-side comparison of the spectra acquired at several positions of both the isolated characteristic edge units in the $50 \mu \mathrm{m}$ silver strips and in the full Koch snowflake fractals. Schematic of Koch snowflake fractals and the charge distribution of an edge mode in the characteristic edge units. Simulated EELS spectra and energy filtered maps of bent silver nanowires analogous to the "V" and "U" characteristic units in the Koch fractals. ADF image and EELS maps of fabricated Koch fractal iteration 3. (PDF)

\section{AUTHOR INFORMATION}

\section{Corresponding Author}

*E-mail: gbotton@mcmaster.ca.

ORCID

Edson P. Bellido: 0000-0003-2444-483X

David Rossouw: 0000-0003-2991-1812

Jérémy Butet: 0000-0001-9598-9074

Olivier J. F. Martin: 0000-0002-9574-3119

Gianluigi A. Botton: 0000-0002-8746-1146

\section{Notes}

The authors declare no competing financial interest.

\section{ACKNOWLEDGMENTS}

E.P.B., D.R., and G.A.B. acknowledge support from NSERC under the Discovery Grant Program. The experimental work was carried out at the Canadian Centre for Electron Microscopy, a National Facility supported by The Canada Foundation for Innovation under the MSI program, NSERC, and McMaster University. O.J.F.M. and G.D.B. acknowledge funding from the Swiss National Science Foundation (project 200020_153662) and from the European Research Council (ERC-2015-AdG-695206 Nanofactory). 


\section{REFERENCES}

(1) Werner, D. H.; Haupt, R. L.; Werner, P. L. Fractal Antenna Engineering: The Theory and Design of Fractal Antenna Arrays. IEEE Antennas Propag. Mag. 1999, 41, 37-58.

(2) Werner, D. H.; Ganguly, S. An Overview of Fractal Antenna Engineering Research. IEEE Antennas Propag. Mag. 2003, 45, 38-57.

(3) Mandelbrot, B. B. The Fractal Geometry of Nature; W. H. Freeman and Company: New York, 1977.

(4) Puente-Baliarda, C.; Romeu, J.; Pous, R.; Cardama, A. On the Behavior of the Sierpinski Multiband Fractal Antenna. IEEE Trans. Antennas Propag. 1998, 46, 517-524.

(5) Baliarda, C.; Romeu, J.; Cardama, A. The Koch Monopole: a Small Fractal Antenna. IEEE Trans. Antennas Propag. 2000, 48, 17731781.

(6) Behera, S.; Vinoy, K. J.; Member, S. Multi-Port Network Approach for the Analysis of Dual Band Fractal Microstrip Antennas. IEEE Trans. Antennas Propag. 2012, 60, 5100-5106.

(7) Omar, A. A. Design of Ultrawideband Coplanar Waveguide-Fed Koch-Fractal Triangular Antenna. Int. J. RF Microwave Comput.-Aided Eng. 2013, 23, 200-207.

(8) Ismahayati, A.; Soh, P. J.; Hadibah, R.; Vandenbosch, G. A. E. Design and Analysis of a Multiband Koch Fractal Monopole Antenna. Proceedings from the IEEE International $R F$ and Microwave Conference, Malaysia, December 12-14, 2011; IEEE: New York, 2011; pp 58-62.

(9) Karim, M. A.; Rahim, M.; Majid, H.; et al. Log Periodic Fractal Koch Antenna for UHF Band Applications. Prog. Electromagn. Res. 2010, 100, 201-218.

(10) Anker, J. N.; Hall, W. P.; Lyandres, O.; Shah, N. C.; Zhao, J.; Van Duyne, R. P. Biosensing with Plasmonic Nanosensors. Nat. Mater. 2008, 7, 442-53.

(11) Kawata, S.; Inouye, Y.; Verma, P. Plasmonics for Near-Field Nano-Imaging and Superlensing. Nat. Photonics 2009, 3, 388-394.

(12) Atwater, H. A.; Polman, A. Plasmonics for Improved Photovoltaic Devices. Nat. Mater. 2010, 9, 205-13.

(13) Neumann, O.; Urban, A. S.; Day, J.; Lal, S.; Nordlander, P.; Halas, N. J. Solar Vapor Generation Enabled by Nanoparticles. ACS Nano 2013, 7, 42-9.

(14) Lal, S.; Clare, S. E.; Halas, N. J. Nanoshell-Enabled Photothermal Cancer Therapy: Impending Clinical Impact. Acc. Chem. Res. 2008, 41, 1842-51.

(15) Mühlschlegel, P.; Eisler, H.-J.; Martin, O. J. F.; Hecht, B.; Pohl, D. W. Resonant Optical Antennas. Science 2005, 308, 1607-1609.

(16) Duan, H.; Hu, H.; Kumar, K.; Shen, Z.; Yang, J. K. W. Direct and Reliable Patterning of Plasmonic Nanostructures with Sub-10-nm Gaps. ACS Nano 2011, 5, 7593-600.

(17) Schnell, M.; García-Etxarri, A.; Huber, a. J.; Crozier, K.; Aizpurua, J.; Hillenbrand, R. Controlling the Near-Field Oscillations of Loaded Plasmonic Nanoantennas. Nat. Photonics 2009, 3, 287-291.

(18) Knebl, D.; Hörl, A.; Trügler, A.; Kern, J.; Krenn, J. R.; Puschnig, P.; Hohenester, U. Gap Plasmonics of Silver Nanocube Dimers. Phys. Rev. B: Condens. Matter Mater. Phys. 2016, 93, 081405.

(19) Ghenuche, P.; Cherukulappurath, S.; Taminiau, T. H.; Van Hulst, N. F.; Quidant, R. Spectroscopic Mode Mapping of Resonant Plasmon Nanoantennas. Phys. Rev. Lett. 2008, 101, 116805.

(20) Fromm, D. P.; Sundaramurthy, A.; James Schuck, P.; Kino, G.; Moerner, W. E. Gap-Dependent Optical Coupling of Single âĂIJBowtie" Nanoantennas Resonant in the Visible. Nano Lett. 2004, 4, 957-961.

(21) Kosako, T.; Kadoya, Y.; Hofmann, H. F. Directional Control of Light by a Nano-Optical Yagi - Uda Antenna. Nat. Photonics 2010, 4, 312-315.

(22) Egerton, R. F. Electron Energy-Loss Spectroscopy in the TEM. Rep. Prog. Phys. 2009, 72, 016502.

(23) Bellido, E. P.; Rossouw, D.; Botton, G. A. Toward $10 \mathrm{meV}$ Electron Energy-Loss Spectroscopy Resolution for Plasmonics. Microsc. Microanal. 2014, 20, 767-78.

(24) Krivanek, O. L.; Lovejoy, T. C.; Dellby, N.; Carpenter, R. W. Monochromated STEM with a $30 \mathrm{meV}$-wide, Atom-Sized Electron Probe. Microscopy 2013, 62, 3-21.
(25) Nelayah, J.; Kociak, M.; Stéphan, O.; García de Abajo, F. J.; Tencé, M.; Henrard, L.; Taverna, D.; Pastoriza-Santos, I.; Liz-Marzán, L. M.; Colliex, C. Mapping Surface Plasmons on a Single Metallic Nanoparticle. Nat. Phys. 2007, 3, 348-353.

(26) Rossouw, D.; Couillard, M.; Vickery, J.; Kumacheva, E.; Botton, G. A. Multipolar Plasmonic Resonances in Silver Nanowire Antennas Imaged with a Subnanometer Electron Probe. Nano Lett. 2011, 11, 1499-1504.

(27) Nicoletti, O.; de la Peña, F.; Leary, R. K.; Holland, D. J.; Ducati, C.; Midgley, P. A. Three-Dimensional Imaging of Localized Surface Plasmon Resonances of Metal Nanoparticles. Nature 2013, 502, 80-4.

(28) Rossouw, D.; Botton, G. A. Plasmonic Response of Bent Silver Nanowires for Nanophotonic Subwavelength Waveguiding. Phys. Rev. Lett. 2013, 110, 066801.

(29) Liang, H.; Zhao, H.; Rossouw, D.; Wang, W.; Xu, H.; Botton, G. A.; Ma, D. Silver Nanorice Structures: Oriented AttachmentDominated Growth, High Environmental Sensitivity, and Real-Space Visualization of Multipolar Resonances. Chem. Mater. 2012, 24, 23392346.

(30) Rossouw, D.; Botton, G. A. Resonant Optical Excitations in Complementary Plasmonic Nanostructures. Opt. Express 2012, 20, 6968-73.

(31) Barrow, S. J.; Rossouw, D.; Funston, A. M.; Botton, G. A.; Mulvaney, P. Mapping Bright and Dark Modes in Gold Nanoparticle Chains Using Electron Energy Loss Spectroscopy. Nano Lett. 2014, 14, 3799-808.

(32) Boudarham, G.; Feth, N.; Myroshnychenko, V.; Linden, S.; García de Abajo, J.; Wegener, M.; Kociak, M. Spectral Imaging of Individual Split-Ring Resonators. Phys. Rev. Lett. 2010, 105, 255501.

(33) Bellido, E. P.; Manjavacas, A.; Zhang, Y.; Cao, Y.; Nordlander, P.; Botton, G. A. Electron Energy-Loss Spectroscopy of Multipolar Edge and Cavity Modes in Silver Nanosquares. ACS Photonics 2016, 3, 428-433.

(34) Bosman, M.; Keast, V. J.; Watanabe, M.; Maaroof, A. I.; Cortie, M. B. Mapping Surface Plasmons at the Nanometre Scale with an Electron Beam. Nanotechnology 2007, 18, 165505.

(35) Schmidt, F. P.; Ditlbacher, H.; Hofer, F.; Krenn, J. R.; Hohenester, U. Morphing a Plasmonic Nanodisk Into a Nanotriangle. Nano Lett. 2014, 14, 4810-5.

(36) Flauraud, V.; Regmi, R.; Winkler, P. M.; Alexander, D. T. L.; Rigneault, H.; van Hulst, N. F.; Garcia-Parajo, M. F.; Wenger, J.; Brugger, J. In-Plane Plasmonic Antenna Arrays with Surface Nanogaps for Giant Fluorescence Enhancement. Nano Lett. 2017, 17, 17031710.

(37) Flauraud, V.; Mastrangeli, M.; Bernasconi, G. D.; Butet, J.; Alexander, D. T. L.; Shahrabi, E.; Martin, O. J. F.; Brugger, J. Nanoscale Topographical Control of Capillary Assembly of Nanoparticles. Nat. Nanotechnol. 2017, 12, 73-80.

(38) Flauraud, V.; Bernasconi, G. D.; Butet, J.; Alexander, D. T. L.; Martin, O. J. F.; Brugger, J. Mode Coupling in Plasmonic Heterodimers Probed with Electron Energy Loss Spectroscopy. ACS Nano 2017, 11, 3485-3495.

(39) Flauraud, V.; Bernasconi, G. D.; Butet, J.; Mastrangeli, M.; Alexander, D. T. L.; Martin, O. J. F.; Brugger, J. Mode Evolution in Strongly Coupled Plasmonic Dolmens Fabricated by Templated Assembly. ACS Photonics 2017, 4, 1661-1668.

(40) Alber, I.; Sigle, W.; Müller, S.; Neumann, R.; Picht, O.; Rauber, M.; van Aken, P. a.; Toimil-Molares, M. E. Visualization of Multipolar Longitudinal and Transversal Surface Plasmon Modes in Nanowire Dimers. ACS Nano 2011, 5, 9845-53.

(41) Bosman, M.; Anstis, G. R.; Keast, V. J.; Clarke, J. D.; Cortie, M. B. Light Splitting in Nanoporous Gold and Silver. ACS Nano 2012, 6, 319-26.

(42) Koh, A. L.; Bao, K.; Khan, I.; Smith, W. E.; Kothleitner, G.; Nordlander, P.; Maier, S. A.; Mccomb, D. W. Electron Energy-Loss Spectroscopy (EELS) of Surface Plasmons in Single Silver Nanoparticles and Dimers: Influence of Beam Damage and Mapping of Dark Modes. ACS Nano 2009, 3, 3015-3022. 
(43) Gottheim, S.; Zhang, H.; Govorov, A. O.; Halas, N. J. Fractal Nanoparticle Plasmonics: The Cayley Tree. ACS Nano 2015, 9, 32843292.

(44) Rosa, L.; Sun, K.; Juodkazis, S. Sierpinski Fractal Plasmonic Nanoantennas. Phys. Status Solidi RRL 2011, 5, 175-177.

(45) Sederberg, S.; Elezzabi, A. Sierpiński Fractal Plasmonic Antenna: a Fractal Abstraction of the Plasmonic Bowtie Antenna. Opt. Express 2011, 19, 10456-10461.

(46) Volpe, G.; Volpe, G.; Quidant, R. Fractal Plasmonics: Subdiffraction Focusing and Broadband Spectral Response by a Sierpinski Nanocarpet. Opt. Express 2011, 19, 3612-3618.

(47) Bao, Y.-J.; Li, H.-M.; Chen, X.-C.; Peng, R.-W.; Wang, M.; Lu, X.; Shao, J.; Ming, N.-B. Tailoring the Resonances of Surface Plasmas on Fractal-Featured Metal Film by Adjusting Aperture Configuration. Appl. Phys. Lett. 2008, 92, 151902.

(48) Aouani, H.; Rahmani, M.; Šípová, H.; Torres, V.; Hegnerová, K.; Beruete, M.; Homola, J.; Hong, M.; Navarro-Cía, M.; Maier, S. A. Plasmonic Nanoantennas for Multispectral Surface-Enhanced Spectroscopies. J. Phys. Chem. C 2013, 117, 18620-18626.

(49) Li, K.; Stockman, M.; Bergman, D. Self-Similar Chain of Metal Nanospheres as an Efficient Nanolens. Phys. Rev. Lett. 2003, 91, 227402.

(50) Aslan, E.; Aslan, E.; Wang, R.; Hong, M. K.; Erramilli, S.; Turkmen, M.; Saracoglu, O. G.; Dal Negro, L. Multispectral CesaroType Fractal Plasmonic Nanoantennas. ACS Photonics 2016, 3, 21022111.

(51) Kenney, M.; Grant, J.; Shah, Y. D.; Escorcia-Carranza, I.; Humphreys, M.; Cumming, D. R. S. Octave-Spanning Broadband Absorption of Terahertz Light Using Metasurface Fractal-Cross Absorbers. ACS Photonics 2017, 4, 2604-2612.

(52) Zhu, L.-H.; Shao, M.-R.; Peng, R.-W.; Fan, R.-H.; Huang, X.-R.; Wang, M. Broadband Absorption and Efficiency Enhancement of an Ultra-Thin Silicon Solar Cell With a Plasmonic Fractal. Opt. Express 2013, 21, A313-A323.

(53) Fang, J.; Wang, D.; DeVault, C. T.; Chung, T.-F.; Chen, Y. P.; Boltasseva, A.; Shalaev, V. M.; Kildishev, A. V. Enhanced Graphene Photodetector with Fractal Metasurface. Nano Lett. 2017, 17, 57-62.

(54) Höppener, C.; Lapin, Z. J.; Bharadwaj, P.; Novotny, L. SelfSimilar Gold-Nanoparticle Antennas for a Cascaded Enhancement of the Optical Field. Phys. Rev. Lett. 2012, 109, 017402.

(55) Navarro-Cia, M.; Maier, S. A. Broad-Band Near-Infrared Plasmonic Nanoantennas for Higher Harmonic Generation. ACS Nano 2012, 6, 3537-3544.

(56) Coluccio, M. L.; Gentile, F.; Das, G.; Nicastri, A.; Perri, A. M.; Candeloro, P.; Perozziello, G.; Proietti Zaccaria, R.; Gongora, J. S. T.; Alrasheed, S.; Fratalocchi, A.; Limongi, T.; Cuda, G.; Di Fabrizio, E. Detection of Single Amino Acid Mutation in Human Breast Cancer by Disordered Plasmonic Self-Similar Chain. Sci. Adv. 2015, 1, e1500487.

(57) Fischer, H.; Martin, O. J. F. Engineering the Optical Response of Plasmonic Nanoantennas. Opt. Express 2008, 16, 9144-9154.

(58) Schmidt, F.-P.; Ditlbacher, H.; Hohenester, U.; Hohenau, A.; Hofer, F.; Krenn, J. R. Universal Dispersion of Surface Plasmons in Flat Nanostructures. Nat. Commun. 2014, 5, 3604.

(59) Campos, A.; Arbouet, A.; Martin, J.; Gerard, D.; Proust, J.; Plain, J.; Kociak, M. Plasmonic Breathing and Edge Modes in Aluminum Nanotriangles. ACS Photonics 2017, 4, 1257-1263.

(60) Bellido, E. P.; Zhang, Y.; Manjavacas, A.; Nordlander, P.; Botton, G. A. Plasmonic Coupling of Multipolar Edge Modes and the Formation of Gap Modes. ACS Photonics 2017, 4, 1558-1565.

(61) Schmidt, F.-P.; Ditlbacher, H.; Hohenau, A.; Hohenester, U.; Hofer, F.; Krenn, J. R. Edge Mode Coupling within a Plasmonic Nanoparticle. Nano Lett. 2016, 16, 5152-5155.

(62) Schmidt, F. P.; Ditlbacher, H.; Hohenester, U.; Hohenau, A.; Hofer, F.; Krenn, J. R. Dark Plasmonic Breathing Modes in Silver Nanodisks. Nano Lett. 2012, 12, 5780-5783.

(63) Prodan, E.; Radloff, C.; Halas, N. J.; Nordlander, P. A Hybridization Model for the Plasmon Response of Complex Nanostructures. Science 2003, 302, 419-22.
(64) Duan, H.; Fernández-Domínguez, A. I.; Bosman, M.; Maier, S. A.; Yang, J. K. W. Nanoplasmonics: Classical Down to the Nanometer Scale. Nano Lett. 2012, 12, 1683-9.

(65) Wen, F.; Zhang, Y.; Gottheim, S.; King, N. S.; Zhang, Y.; Nordlander, P.; Halas, N. J. Charge Transfer Plasmons: Optical Frequency Conductances and Tunable Infrared Resonance. ACS Nano 2015, 9, 6428-6435.

(66) Leapman, R.; Swyt, C. Separation of Overlapping Core Edges in Electron Energy Loss Spectra by Multiple-Least-Squares Fitting. Ultramicroscopy 1988, 26, 393-403.

(67) Mazzucco, S.; Geuquet, N.; Ye, J.; Stéphan, O.; Van Roy, W.; Van Dorpe, P.; Henrard, L.; Kociak, M. Ultralocal Modification of Surface Plasmons Properties in Silver Nanocubes. Nano Lett. 2012, 12, 1288-94.

(68) Rodríguez-Fortuño, F. J.; García-Meca, C.; Ortuño, R.; Martí, J.; Martínez, A. Modeling High-Order Plasmon Resonances of a UShaped Nanowire Used to Build a Negative-Index Metamaterial. Phys. Rev. B: Condens. Matter Mater. Phys. 2009, 79, 075103.

(69) Bernasconi, G. D.; Butet, J.; Flauraud, V.; Alexander, D.; Brugger, J.; Martin, O. J. F. Where Does Energy Go in Electron Energy Loss Spectroscopy of Nanostructures? ACS Photonics 2017, 4, 156164.

(70) Bernasconi, G. D.; Butet, J.; Martin, O. J. F. Mode Analysis of Second-Harmonic Generation in Plasmonic Nanostructures. J. Opt. Soc. Am. B 2016, 33, 768-779.

(71) Hohenester, U.; Trügler, A. MNPBEM - A Matlab Toolbox for the Simulation of Plasmonic Nanoparticles. Comput. Phys. Commun. 2012, 183, 370-381.

(72) Hohenester, U. Simulating Electron Energy Loss Spectroscopy with the MNPBEM Toolbox. Comput. Phys. Commun. 2014, 185, $1177-1187$.

(73) Babar, S.; Weaver, J. H. Optical Constants of $\mathrm{Cu}, \mathrm{Ag}$, and $\mathrm{Au}$ Revisited. Appl. Opt. 2015, 54, 477. 